\title{
Fabrication of carbon nanotube-reinforced mortar specimens: evaluation of mechanical and pressure-sensitive properties
}

\author{
Evangelia K. Karaxi, Irene A. Kanellopoulou, Anna Karatza, Ioannis A. \\ Kartsonakis, Costas A. Charitidis*
}

National Technical University of Athens, School of Chemical Engineering, RNANO Lab - Laboratory of Advanced, Composite, Nanomaterials and Nanotechnology, 9 Heroon Polytechniou str., Zografou Campus, 15773, Athens, Greece

\begin{abstract}
Carbon-based nanomaterials are promising reinforcing elements for the development of "smart" self-sensing cementitious composites due to their exceptional mechanical and electrical properties. Significant research efforts have been committed on the synthesis of cement-based composite materials reinforced with carbonaceous nanostructures, covering every aspect of the production process (type of nanomaterial, mixing process, electrode type, measurement methods etc.). In this study, the aim is to develop a well-defined repeatable procedure for the fabrication as well as the evaluation of pressure-sensitive properties of intrinsically self-sensing cementitious composites incorporating carbon- based nanomaterials. Highly functionalized multi-walled carbon nanotubes with increased dispersibility in polar media were used in the development of advanced reinforced mortar specimens which increased their mechanical properties and provided repeatable pressure-sensitive properties.
\end{abstract}

\section{Introduction}

Concrete is one of the most used resources in the world, since most of the infrastructures are built with it. However, due to the deterioration of cement-based materials as a result of their interaction with their service environment, significant and costly efforts are needed to restore the failing structures to their safe state. In addition to embedded sensors for on-line measurement and monitoring of mechanical stresses, strains and accelerations in the case of dynamically loaded structures, significant scientific research is focused on the development of intrinsically self-sensing concrete. This intelligent multi-phase material could help us built "smart" infrastructures exhibiting structural health monitoring abilities and improved service-life, safety and reliability.

The self-monitoring concrete can be fabricated by the incorporation of intrinsically conductive fillers such as carbon fibers $(\mathrm{CF})$, carbon nanotubes or nanofibers (CNT/CNF) and metallic powered materials (e.g. nickel powder). The aim is to successfully disperse these functional fillers in the cementitious matrix so as to form an extensive conductive network 
and consequently increase its ability to sense the formation of cracks in itself while still maintaining or even improving its mechanical properties [1].

Although the research on self-sensing concrete emerged nearly two decades ago, there are still many critical challenges which have to be overcome before its deployment. The selfsensing concrete is a complex composite material which incorporates a variety of components, phases and scales. Its fabrication in a repeatable way is still a challenge, especially when the incorporation of nanoscale additives is in need, due to the lack of a standardized method for its design and realization as well as for the evaluation of additives dispersion in concrete matrix. On the other hand, the sensing signal is highly depended on many measurement parameters such as the electrode and circuit design and its processing method.

The aim of this work is twofold: i) the development of intrinsically self-sensing cementbased composites ii) the deployment of tailored reinforced bulk cementitious matrices with added value functionalities capable to extend the service life of concrete infrastructures. Multi-walled CNT (MWCNT) were synthesized via the thermally assisted chemical vapour deposition (T-CVD) method using the supported catalyst approach. Much effort was focused on the development of a standard procedure for the functionalization of the as- received CNTs with high number of oxygen-containing groups. The ultimate target is to improve their dispersibility in polar solvents and consequently in cement-based materials so as to overcome the poor dispersion issues of the highly hydrophobic carbon-based nanofillers in cementitious matrices without the addition of dispersants and surfactants which dramatically decrease the bulk electrical conductivity of the final composite [2]. After the evaluation of the conductive fillers before and after their surface functionalization, the MWCNT-reinforced mortar samples were fabricated in triplicates. Their morphology, mechanical properties and selfsensing ability were then evaluated as a function of the MWCNT concentration.

\section{Experimental}

\subsection{Materials}

All chemicals were of analytical reagent grade. Acetylene (Air Liquide), $\mathrm{Fe}\left(\mathrm{NO}_{3}\right)_{3} \cdot 9 \mathrm{H}_{2} \mathrm{O}$ (Aldrich, St. Louis, USA), zeolite (Alfa Aesar; particle size $\sim 1 \mu \mathrm{m}$; specific surface area 975 $\mathrm{m}^{2} / \mathrm{g}$ ). Portland Cement CEM I $52.5 \mathrm{~N}$ was used as cementitious material.

\subsection{Synthesis of MWCNTs}

Synthesis of MWCNTs was performed according to our previous work [3]. Fe/zeolite particles were used as catalyst for the CNTs synthesis. The catalyst was synthesized by impregnation method using zeolite powder as supporting material and $\mathrm{Fe}\left(\mathrm{NO}_{3}\right)_{3}$ as metal precursor. Firstly, $4 \mathrm{~g}$ zeolite and $12 \mathrm{~g} \mathrm{Fe}\left(\mathrm{NO}_{3}\right)_{3}$ were separately dispersed in $200 \mathrm{ml}$ of distilled $\mathrm{H}_{2} \mathrm{O}$. The amount of the iron salt was suitably calculated to obtain catalyst with a nominal Fe content $20 \mathrm{wt} \%$. Then, the obtained zeolite dispersion were stirred at $70{ }^{\circ} \mathrm{C}$ during which the $\mathrm{Fe}\left(\mathrm{NO}_{3}\right)_{3}$ solution was gradually added to the mixture in a period of $30 \mathrm{~min}$. The resulting dispersion was centrifuged, the supernatant solutions were discarded, and then the particles were re-suspended in water using a sonicator. This process was repeated three times and the purified powders were dried at $100{ }^{\circ} \mathrm{C}$. The produced material was thermally treated at $500{ }^{\circ} \mathrm{C}$ in an oven under nitrogen flow for $1 \mathrm{~h}$. A T-CVD reactor that consists of a horizontal quartz tube was used to synthesize MWCNTs. The catalyst 
particles (Fe/zeolite) were placed on a silicon substrate which was located inside the quartz tube, in the middle of the isothermal zone of the reactor. Firstly, a constant argon flow rate $(200 \mathrm{~mL} / \mathrm{min}$ ) was passed through the quartz tube to remove the air from the system and then the reactor was heated at $800{ }^{\circ} \mathrm{C}$ under argon flow. Subsequently, the argon was replaced by the $350 \mathrm{~mL} / \mathrm{min}$ flow of mixture of acetylene/argon (40:60) and the synthesis was carried out at the same temperature for $1 \mathrm{~h}$; where pyrolysis of the acetylene took place and carbonaceous materials were deposited on the silicon substrate. When the reaction was completed, the raw entangled MWCNT products were cooled down to room temperature in argon atmosphere. Due to the impurities contained in the final product (graphite, amorphous carbon, fullerenes and iron particles derived from the catalytic particles), a standardized purification process was developed so as to remove all these unnecessary products which is one of the most fundamental challenges in carbon-based materials production. The product was first calcined at $400{ }^{\circ} \mathrm{C}$ to remove the amorphous carbon, then it was treated with an alkaline and an acidic solution $(1 \mathrm{M} \mathrm{NaOH}$ and $3 \mathrm{M} \mathrm{HCl}$ respectively) to remove the supporting particles and the metallic iron particles, respectively, and the final step included washings with distilled water. For further functionalization and to activate the MWCNT surface with oxygen-containing groups an acid solution mixture of $\mathrm{HNO}_{3}: \mathrm{H}_{2} \mathrm{SO}_{4}(1: 3 \mathrm{v} / \mathrm{v})$ was used. Before the treatment MWCNTs milling was necessary. The MWCNT/acid mixture $(0.15 \mathrm{~g}$ MWCNTs $/ 10 \mathrm{ml}$ acid solution) was stirred for $5 \mathrm{~h}$ at room temperature. The treatment was followed by quenching in ice and then neutralization by $\mathrm{NaOH}$ water solution. The suspension was filtered and the black powder deposited on the filter was washed with distilled water, then with ethanol and acetone and dried in oven. All the above-mentioned samples were functionalized with carboxylic groups to enhance their hydrophilic properties and the dispersion in water-based mixtures.

\subsection{Mortar/MWCNT composites fabrication and testing}

Portland Cement CEM I $52.5 \mathrm{~N}$ was used as cementitious material; its physical properties and chemical composition are summarized in Table 1. Mortar specimens reinforced with CNTs were synthesised and characterized with respect to their i) self-sensing capability (piezoresistivity tests) and ii) macro mechanical integrity by means of flexural strength (EN 196-1) and compressive strength (EN 196-1) tests. Regarding the piezoresistivity tests, prismatic test samples $40 \mathrm{~mm}$ x $40 \mathrm{~mm}$ x $40 \mathrm{~mm}$ in size with embedded stainless steel electrodes were fabricated (in triplicates). The flexural and compressive strength measurements were conducted on prismatic test samples $40 \mathrm{~mm}$ x $40 \mathrm{~mm}$ x $160 \mathrm{~mm}$ in size fabricated (in triplicates). MWCNTs were incorporated into the aforementioned mixture as additives at $0.02 \%, 0.05 \%, 0.1 \%, 0.2 \%, 0.5 \%$ and $1.0 \%$ by weight of cement (bwoc). Prior to the preparation of the composite samples the surface of the carbon-based materials was functionalized by introducing polar functionalized groups such as hydroxyl $(-\mathrm{OH})$, carboxyl $(-\mathrm{COOH})$ and carbonyl $(=\mathrm{CO})$ groups. This modification promotes the wettability of the MWCNTs and the formation of covalent bonding between the MWCNT-surface and the calcium-silicate-hydrate phases formed after 28 days of hydration, as reported early in literature [4]. The mortar was prepared by mechanical mixing and was compacted in a teflon mould using a jolting apparatus. The specimens were immediately moulded after the preparation of the mortar. With the mould and hopper firmly clamped to the jolting table, the first of two layers of mortar (each about $300 \mathrm{~g}$ ) were introduced in one or more increments, using a suitable scoop, into each of the mould compartments, directly from the mixing bowl. The layer was uniformly spread using the larger spreader. Then, the first mortar layer was compacted with 60 jolts. After that, the second layer of mortar was introduced, leveled with the smaller spreader and compacted with 60 jolts. Then, a glass plate of $6 \mathrm{~mm}$ thickness was placed on the mould for $24 \mathrm{~h}$. After 24 hours, the specimens 
were demolded and submerged into distilled water for 28 days. Regarding the piezoresistive measurements, the same procedure was followed. The stainless-steel electrodes were embedded and the mortar was further compacted with 10 jolts.

Table 1 Chemical composition and physical properties of CEM I $52.5 \mathrm{~N}$.

\begin{tabular}{ll}
\hline Composition \% (mass) & Cement \\
\hline $\mathrm{SiO}_{2}$ & 19.47 \\
$\mathrm{Al}_{2} \mathrm{O}_{3}$ & 4.75 \\
$\mathrm{Fe}_{2} \mathrm{O}_{3}$ & 3.43 \\
$\mathrm{CaO}$ & 63.16 \\
$\mathrm{MgO}$ & 1.43 \\
$\mathrm{SO}_{3}$ & 2.68 \\
$\mathrm{Na}_{2} \mathrm{O}$ & 0.28 \\
$\mathrm{~K}_{2} \mathrm{O}$ & 0.62 \\
Loss on Ignition & 3.26 \\
Specific surface area $\left(\mathrm{cm}^{2} / \mathrm{g}\right)$ & 3635 \\
\hline
\end{tabular}

\subsection{Instrumental analysis}

Scanning Electron Microscopy (SEM) was utilized to characterize the morphology of the MWCNTs prior to their incorporation in the cementitious matrix using a Phillips Quanta Inspect (FEI Company) microscope, QUANTA 200 with W (tungsten) filament $25 \mathrm{kV}$ equipped with EDAX GENESIS (AMETEX PROCESS \& ANALYTICAL INSTRUMENTS).

The macro mechanical integrity was investigated by means of flexural strength using a material testing machine to apply loads (Instron 1121) with a displacement rate (speed) of 1 $\mathrm{mm} / \mathrm{min}$ and by means of compressive strength using an INSTRON 300DX-B1-C4-G6C with a displacement rate (speed) of $5 \mathrm{~mm} / \mathrm{min}$.

The aim of relative resistance measurement using four-probe method under cyclic loading was to monitor the material's sensing capability during a loading-unloading procedure. For the electrical measurements, a material testing machine was used to apply loads (Instron 1121) and a Keithley Source-Meter 2400 was used. At each measurement a fixed direct current (DC) of $1 \mathrm{~mA}$ was applied to the outer two electrical contacts while the voltage drop between the inner electrical contacts was measured. The displacement rate (speed) was 1 $\mathrm{mm} / \mathrm{min}$, the cyclic load ranged from 0.2 to $9.5 \mathrm{kN}$ (a pre-stress was applied equal to $200 \mathrm{~N}$ ) and a laser extensometer Fielder Optoelektronik K-100 was used to accurately measure the induced displacement on the composites during the multiple loading cycles. The corresponding voltage signal was continuously collected. Then the relative change of the resistivity $\left(\Delta \rho / \rho_{0}\right)$ as a function of time was calculated, where $\rho_{0}$ is the initial electrical resistivity of specimens without compressive loading at the beginning of the experiment. The results can be depicted either as a function of load or as a function of stress. In order to eliminate the effect of electric polarization and depolarization of cement-based materials on compressive sensitivity testing results, enough time was left to pass till the voltage value is stable. Due to the fact that the resistivity is independent of the area of voltage pole and the mesh size of the electrode, the area of the voltage pole can be decreased and the mesh size can be increased to avoid any influence on the mechanical integrity of the samples. 


\section{Results and Discussion}

\subsection{Instrumental Analysis}

SEM: Figure 1 depicts SEM image of the final purified and functionalized MWCNTs. The purity of the produced materials in terms of their morphology and chemical composition was confirmed through the SEM images and the Energy Dispersive X-Ray Spectroscopy spectra. The SEM characterization confirmed the successful synthesis of MWCNT with the aforementioned experimental procedure and from the EDS analysis the only chemical elements detected were $\mathrm{C}$ and $\mathrm{O}$, as the $\mathrm{Fe}$ and zeolite particles were rejected through the purification process (Table 2).

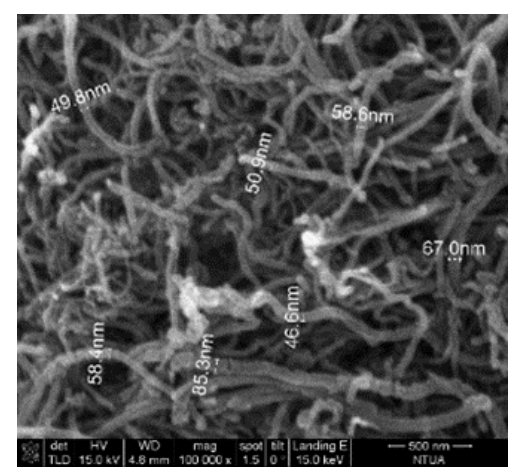

Fig. 1. SEM image MWCNT after the purification and functionalization process (x 100.000)

Table 2. SEM-EDS analysis for carbon and oxygen and silicon in MWCNT sample.

\begin{tabular}{|c|c|c|c|c|}
\hline \multirow{2}{*}{ Sample } & \multicolumn{2}{|c|}{ C } & \multicolumn{2}{c|}{ O } \\
\cline { 2 - 5 } & wt \% & at \% & wt \% & at \% \\
\hline MWCNT & 96.18 & 97.01 & 3.82 & 2.89 \\
\hline
\end{tabular}

The effective $-\mathrm{COOH}$ functionalization was confirmed through TGA analysis in nitrogen atmosphere $\left(50 \mathrm{~mL} / \mathrm{min}\right.$ gas flow) in a temperature range of $25-800{ }^{\circ} \mathrm{C}$ with a heating rate of $1{ }^{\circ} \mathrm{C} / \mathrm{min}$. The diagram is depicted in Figure 2a. Oxygen-containing functional groups decompose under higher temperatures, according to the following equations

$$
\begin{aligned}
& \mathrm{RCOOH} \rightarrow \mathrm{RH}+\mathrm{CO}_{2} \\
& \mathrm{RCOH} \rightarrow \mathrm{RH}+\mathrm{CO} \\
& \mathrm{RCOOR} \rightarrow \mathrm{RR}+\mathrm{CO}_{2}
\end{aligned}
$$

The mass loss in the region from room temperature up to $150{ }^{\circ} \mathrm{C}$ is related to the release of physisorbed water. The carboxylic groups can be observed in the range from 150 to $300{ }^{\circ} \mathrm{C}$. Further mass loss can be attributed to the decomposition of carbonyl groups, which can be observed at temperatures higher than $700{ }^{\circ} \mathrm{C}$. However, the determination of decomposition products is more difficult at higher temperatures. In the examined MWCNT sample, a mass reduction (1.89\%) from 180 until $380{ }^{\circ} \mathrm{C}$ is attributed to the decarboxylation of the 
carboxylic groups present on the MWCNT walls. Thermal degradation in the range between 400 and $700{ }^{\circ} \mathrm{C}$ may be explained by the elimination of hydroxyl functionalities attached to the MWCNT walls. Finally, at temperatures higher that $750{ }^{\circ} \mathrm{C}$, the observed degradation corresponds to the thermal oxidation of the remaining disordered carbon structures. This phenomenon is usually observed after the acid-treatment of the MWCNT which creates a number of defects to the graphene cylinders.

Information about the oxidative process/thermal stability and purity was obtained via TGA analysis in oxygen atmosphere $\left(50 \mathrm{~mL} / \mathrm{min}\right.$ gas flow) in a temperature range of $25-800{ }^{\circ} \mathrm{C}$ with a heating rate of $1{ }^{\circ} \mathrm{C} / \mathrm{min}$ (Figure 2b). It can be seen that the oxidative temperature was at $600{ }^{\circ} \mathrm{C}$.

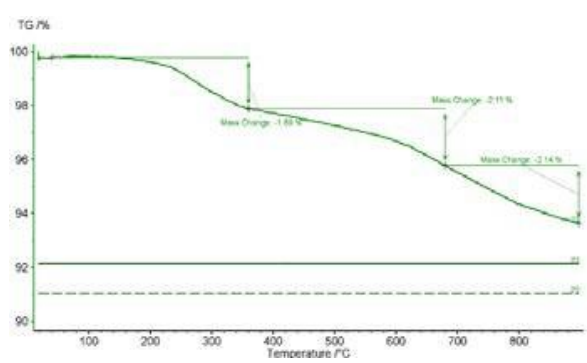

Fig. 2a. TG analysis of acid-treated MWCNT in inert atmosphere.

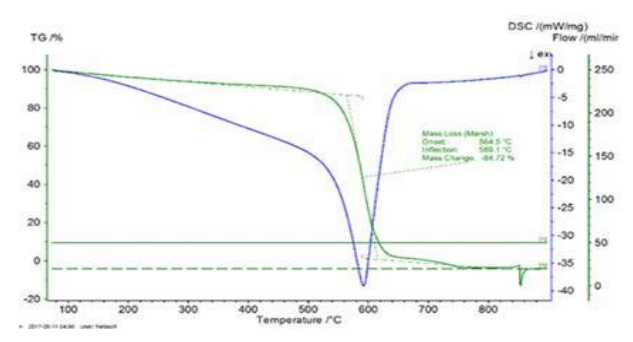

Fig. 2b. TG analysis of acid-treated MWCNT in oxidative atmosphere

The X-ray photoelectron spectroscopy (XPS) of acid-treated MWCNT with - $\mathrm{COOH}$ functional groups (Table 2) further confirmed that the oxygen-containing groups have been successfully introduced (13.4\% at.) onto MWCNTs sidewalls which facilitate the exfoliation of CNT bundles.

Table 2. XPS analysis of pristine and acid-treated MWCNT.

\begin{tabular}{|c|c|c|}
\hline Chemical Bond & Pristine MWCNTs & Functionalized MWCNTs with -COOH \\
\hline $\mathrm{C}-\mathrm{C}\left(\mathrm{sp}^{3}\right)$ & $7.6 \%$ at. & $15.9 \%$ at. \\
\hline $\mathrm{C}=\mathrm{C}\left(\mathrm{sp}^{2}\right)$ & $81.5 \%$ at. & $66.2 \%$ at. \\
\hline $\mathrm{C}-\mathrm{O}$ & $2.8 \%$ at. & $6.3 \%$ at. \\
\hline $\mathrm{C}=\mathrm{O}$ & $1.6 \%$ at. & $2.6 \%$ at. \\
\hline $\mathrm{COOH}$ & - & $4.5 \%$ at. \\
\hline
\end{tabular}

Results of the piezoresistive response of $0.2 \%$ MWCNTs mortar composites are shown in Figure 3. The graph reflects the cycles of loading-unloading performed and the corresponding change in resistivity. The fractional change in resistivity $\left(\Delta \rho / \rho_{0}, \%\right)$ of the composite fabricated with 0.2 wt.\% MWNTs revealed that the resistance changes also correspond to the stress load levels. It was observed that resistivity decreased during loading and increased during unloading. It was also indicated that the response of the electrical resistance of this composite to compressive stress $\sigma$ was regular under repeated compressive loading. The most important factor contributing to this change of the resistance values was the contact resistance. Compressive loading results to a decrement of the gap at the contact area, where electrical tunnelling takes place and thus decreases the contact resistance. According to these results, it can be seen that the response of the electrical resistance of selfsensing MWCNT/mortar composite to compressive stress was reversible, which means that the self-sensing MWCNT/mortar composite has a sensing capability. 

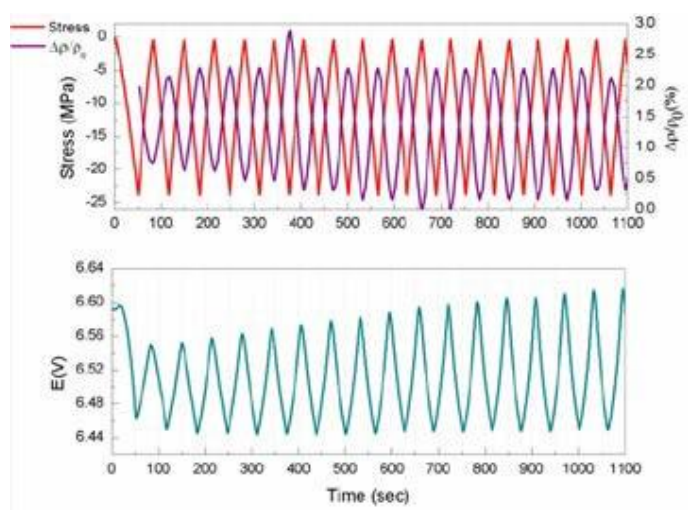

Fig. 3 Piezoresistive diagram of mortar/ CNTs $0.2 \%$ bwoc.

In Figure 4a an increment on the flexural strength of the composites by $43 \%$ in the case of $0.05 \%$ MWCNT bwoc can be observed most probably due to the crack bridging effect of the MWCNT. The MWCNT used in this work exhibited enhanced affinity with the cementitious matrix as in comparison to literature in many cases much higher concentrations reaching $0.25 \%$ MWCNT bwoc are needed to improve the mechanical properties of the composites $[5,6]$. On the other hand, the test results derived from the compression tests demonstrated an enhancement of $5 \%$ on the mortar compressive strength due to the integration of 0.2 MWCNTs into the mix which was not as significant as the enhancement in the flexural strength (Figure 4b),

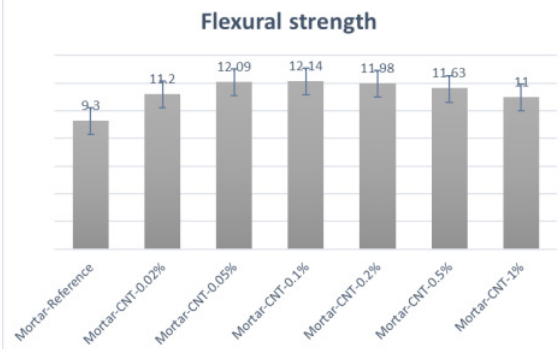

Fig. 4a Flexural strength of prismatic mortar specimens reinforced with MWCNT.

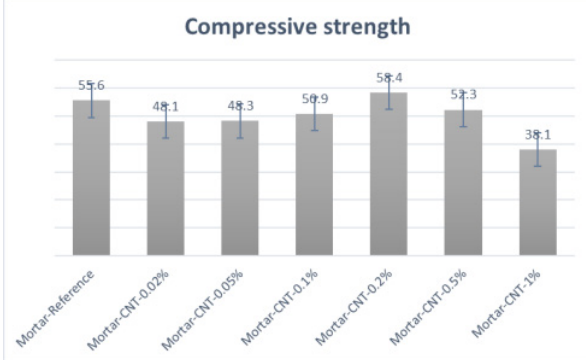

Fig. 4b. Compressive strength of prismatic mortar specimens reinforced with MWCNT.

The poor mechanical properties observed after the addition of higher amounts of carboxylfunctionalized MWCNTs can be related to an inhibition of the mortar hydration reactions and the formation of the corresponding calcium silicate phases (C-S-H) phases [6]. More specifically, tobermorite gel, which is formed during the hydration reactions of the C-S-H phases such as $\mathrm{C}_{3} \mathrm{~S}\left(3 \mathrm{CaO} \cdot \mathrm{SiO}_{2}\right)$ and $\mathrm{C}_{2} \mathrm{~S}\left(2 \mathrm{CaO} \cdot \mathrm{SiO}_{2}\right)$, employs a significant enhancement on the mechanical strength of cement-based composites, as demonstrated in literature [7]. We assume that, due to the increased hydrophilic nature of the MWCNTs' surface, a considerable amount of water is absorbed which hampers the cement paste hydration and consequently the mechanical performance of the fabricated composites. In future experiments, a new series of composites will be developed with the addition of an excess of water (increased water/cement ratio) so as to investigate the influence of this parameter on their mechanical performance when adding higher amounts of acid-treated MWCNTs. Additionally, the samples will be evaluated through Thermogravimetric Analysis to determine the weight percentage of the crystalline phases as a function of the MWCNTs and water amount during their thermal decomposition.

\section{Conclusions}


Within this work, a well-defined procedure was developed for the modification of MWCNT surface with reactive groups, the addition of the nanoscale additives into the mortar mixture as well as the evaluation of the composites' sensing capability. The flexural strength of the mortar/MWCNT samples was increased by $43 \%(0.05 \%$ bwoc $)$ due to the crack bridging effect of the fibrous carbonaceous material. The electrical resistance of the resulting composite specimens exhibited reversible response to the multiple loading cycles revealing their pressure-sensitive capabilities without the need of adding dispersing agents that usually negatively affect the sensing ability of such complex composite systems.

These results are part of a project that has received funding from the European Union's Horizon 2020 research and innovation program under grant agreement No685445 (LORCENIS).

\section{References}

1. Han, B., S. Ding, and X. Yu, Measurement, 59: pp. 110-128 (2015).

2. J.A. Epaarachchi, et al., Fourth International Conference on Smart Materials and Nanotechnology in Engineering (2013).

3. A.-F. A. Trompeta et al. Manufacturing Review, 4, pp. 7-15 (2017).

4. G.Y Li, P.M. Wang, and X. Zhao, Carbon,. 43, pp. 1239-1245, (2005).

5. Mohsen, M.O., et al., Construction and Building Materials,. 150, pp. 395-403, (2017).

6. Jang, S. H., S. Kawashima, and H. Yin. Materials (Basel) 9, (2016).

7. Dweck, J., et al., Journal of Thermal Analysis and Calorimetry, 69: pp. 179-186 (2002). 